\title{
EFECTO DE LA COTIZACIÓN INTERNACIONAL DEL COBRE EN LA RECAUDACIÓN DEL IMPUESTO A LA RENTA EMPRESARIAL DEL SECTOR MINERO
}

\author{
EFFECT OF THE INTERNATIONAL COPPER PRICE'S QUOTE ON CORPORATE INCOME \\ TAX COLLECTION IN THE MINING SECTOR
}

Ronald Studer Lévano Huamaccto Ernst \& Young Consultores SRL

Lima, Perú

ORCID: https://orcid.org/0000-0002-5888-8300 Autor para correspondencia: ronald.levano@pe.ey.com

Bill Frank Oscanoa Ponce Ernst \& Young Consultores SRL Lima, Perú

ORCID: https://orcid.org/0000-0002-8781-9363 Correo electrónico: bill.oscanoa@pe.ey.com

Edmundo Rafael Casavilca Maldonado Universidad Nacional Mayor de San Marcos Lima, Perú

ORCID: https://orcid.org/0000-0001-8625-9811

Correo electrónico: ecasavilcam@gmail.com

[Recibido: 29/09/2021 Aceptado: 07/12/2021 Publicado: 29/12/2021]

\section{RESUMEN}

Objetivo: Establecer el efecto de la cotización internacional del precio del cobre en la recaudación del impuesto a la renta empresarial (RIRE) del sector minero peruano correspondiente al periodo 2004-2020. Método: El diseño fue no experimental, retrospectivo, transversal y analítico, con enfoque cuantitativo, de tipo descriptivo, correlacional y explicativo. En ese sentido, se recurrió a la estadística inferencial, aplicando la prueba de normalidad de Shapiro Wilk, así como a pruebas de hipótesis para determinar la relación entre las variables de estudio y el análisis de regresión para establecer el impacto entre las mismas. Resultado: Existe una relación positiva entre la cotización internacional del precio del cobre y la RIRE del sector minero peruano, con un grado de correlación de $86,9 \%$ y un coeficiente de determinación de $75,52 \%$, a un nivel de confianza de $99 \%$. Conclusión: La cotización internacional del precio del cobre sí afectó en la RIRE del sector minero peruano en el periodo 2004-2020.

Palabras clave: Cotización internacional; precio del cobre; impuesto a la renta; sector minero.

\begin{abstract}
Objective: Establish the effect of the international copper price's quote on the corporate income tax collection (RIRE) of the Peruvian mining sector for the period 2004-2020. Method: The design was non-experimental, retrospective, cross-sectional and analytical, with a quantitative, descriptive, correlational, and explanatory approach. In this sense, inferential statistics were used, applying the Shapiro Wilk normality test, as well as hypothesis tests to determine the relationship between the study variables and regression analysis to establish the impact between them. Result: There is a positive relationship between the international copper price and the corporate income tax collection of the Peruvian mining sector, with a correlation degree of $86,9 \%$ and a determination coefficient of $75,52 \%$, at a confidence level of $99 \%$. Conclusion: The international copper price did affect the corporate income tax collection of the Peruvian mining sector in the period 2004-2020.
\end{abstract}

Keywords: International quotation; copper price; income tax; Peruvian mining sector.

(c) Los autores. Este artículo es publicado por la revista Quipukamayoc de la Facultad de Ciencias Contables, Universidad Nacional Mayor de San Marcos. Este es un artículo de acceso abierto, distribuido bajo los términos de la licencia Creative Commons Atribución 4.0 Internacional (CC BY 4.0) [https://creativecommons.org/licenses/ by/4.0/deed.es] que permite el uso, distribución y reproducción en cualquier medio, siempre que la obra original sea debidamente citada de su fuente original. 


\section{INTRODUCCIÓN}

Durante la última década, la actividad minera ha sido uno de los motores más importantes del crecimiento económico en el país, "llegando a representar el $18,1 \%$ de la recaudación tributaria total entre el periodo 2006 y el 2010" (Peñaranda, 2019, p. 2). Asimismo, de acuerdo con la Confederación Nacional de Instituciones Empresariales Privadas (2021) el aporte de la minería formal en el año 2020 fue de $14 \%$, por lo que es importante resaltar que la minería "impacta en la generación de empleo directo e indirecto, contribuye a la recaudación fiscal, dinamiza la economía, descentraliza la actividad productiva y aporta el desarrollo sostenible" (párr. 2).

En efecto, "el sector minero es responsable de 10\% del PBI, de $60 \%$ de las exportaciones, de $16 \%$ de la inversión privada y $19 \%$ de los tributos pagados por empresas, según la Sociedad Nacional de Minería, Petróleo y Energía (SNMPE)” (Gestión, 2020, párr. 3). Por otro lado, también es importante resaltar lo siguiente:

La minería es una de las actividades que más aporta a la recaudación fiscal. El régimen tributario peruano se ubica $3,8 \%$ por encima del promedio de los principales países mineros y las empresas mineras peruanas enfrentan una carga fiscal equivalente al 47,07\%. (Instituto Peruano de Economía, 2021, párr. 10)

Ahora bien, el incremento significativo del producto bruto interno ( $\mathrm{PBl}$ ) minero ha ido a la par con el aumento de las exportaciones, inversiones, producción y también del potencial impacto de la volatilidad de los resultados de los precios internacionales de minerales como el cobre, oro, zinc, plomo, plata, estaño, hierro, molibdeno y otros. Por ello, se debe tomar en cuenta que los resultados de la volatilidad de los precios de los minerales se explican por la interacción de la oferta y demanda en los mercados internacionales, motivo por el cual resulta importante evaluar el efecto de la cotización internacional del precio del cobre en la recaudación del impuesto a la renta empresarial del sector minero peruano.

Siendo así, durante los últimos decenios, el Perú se ha dedicado a extraer y exportar minerales como materia prima sin dar un mayor valor agregado, esto juega un papel adverso para la economía nacional. Por ello, los efectos positivos y negativos que puedan generarse en los mercados internacionales terminan impactando directamente en la economía nacional; por lo que, de seguir con dicha tendencia, resulta importante comprender la implicancia de la volatilidad de los precios internacionales de los minerales, en específico y siendo el más relevante el cobre, mineral que ha cobrado mayor representatividad en las últimas décadas en la recaudación del impuesto a la renta empresarial (en adelante RIRE).

De esta manera, De la Cuba y Ferreyra (2020) aseveran que "en los últimos doce años se identifican tres periodos de caída severa en el precio del cobre: la crisis financiera global (2008-2009), la crisis bursátil china (2015-2016) y la reciente pandemia (enero-junio 2020)" (p. 32). Por otro lado, señalan que los factores que ayudan a explicar la caída menos profunda y la recuperación más rápida del mercado internacional del precio de minerales durante la pandemia resultan ser:

(i) el mayor impacto que ha tenido la pandemia sobre el sector servicios, (ii) las restricciones en el mercado de concentrados como en el de cobre reciclado- asociadas en gran medida al impacto del COVID-19, (iii) la recuperación más rápida de la demanda no comercial, y (iv) la menor apreciación del dólar (por la aplicación más rápida de estímulos monetarios por parte de la Fed). (De la Cuba y Ferreyra, 2020, p. 37)

Lo mencionado anteriormente permite explicar por qué diciembre de 2020 se caracterizó por presentar resultados favorables en las cotizaciones promedio de los principales metales en virtud de las variaciones positivas. De esta manera, según datos del Ministerio de Energía y Minas (2020) "en contraste a diciembre de 2019, los principales productos metálicos mejoraron su cotización, dentro de los cuales el cobre registró un incremento de $+27,6 \% "$ (p. 13).

Asimismo, los factores que influyeron en la cotización del metal rojo durante el último mes de 2020 fueron:

(i) Las actividades fabriles en China se aceleraron al ritmo más rápido en una década, lo cual provocó un incremento en la demanda de cobre en el mercado mundial; (ii) la aprobación del paquete de estímulo fiscal estadounidense generó expectativas de una sólida y dinámica recuperación económica en el 2021, siendo un signo positivo para el cobre; (iii) el lanzamiento de vacunas contra el Covid-19 [sic] avivó las expectativas de una recuperación económica mundial y, (iv) las existencias de cobre en depósitos a nivel mundial descendieron en casi $30 \%$ desde mediados de octubre debido a la demanda de China, ocasionando escasez de este mineral y propiciando el incremento de su precio. (Ministerio de Energía y Minas, 2020, pp. 13-14)

En consecuencia, lo indicado anteriormente resulta importante para realizar el análisis relacionado con la evolución y comportamiento de la RIRE del sector minero peruano durante el periodo 2004-2020. 
Tal como se aprecia en la Figura 1, la RIRE del sector minero no muestra resultados favorables debido a su comportamiento irregular durante las últimas décadas, lo que resulta perjudicial no solo para el crecimiento económico del sector minero, sino para el país, por su significativo aporte al PBI nacional.

Por lo tanto, bajo la situación identificada con relación a la RIRE del sector minero peruano, resulta relevante identificar qué variables son determinantes, ya que estas tienen implicancia en el comportamiento irregular de la recaudación fiscal. En atención a ello, se observa que la cotización internacional del precio del cobre mantiene un comportamiento semejante a la recaudación tributaria aludida, tal como se observa en la Figura 2.

Por otro lado, la teoría económica del orden construido y espontáneo permiten comprender las variables de estudio: la RIRE y la cotización internacional del precio de cobre.

\begin{tabular}{|l|c|}
\hline 2004 & 1,741 \\
2005 & 3,123 \\
2006 & 7,731 \\
2007 & 10,761 \\
2008 & 8,985 \\
2009 & 4,859 \\
2010 & 8,132 \\
2011 & 11,258 \\
2012 & 10,633 \\
2013 & 7,181 \\
2014 & 7,430 \\
2015 & 4,349 \\
2016 & 4,307 \\
2017 & 6,990 \\
2018 & 9,835 \\
2019 & 8,001 \\
2020 & 5,908 \\
\hline
\end{tabular}

Figura 1. Impuesto a la renta empresarial del sector minero en el periodo 2004-2020

Fuente: Elaboración propia sobre la base de la Superintendencia Nacional de Aduanas y de Administración Tributaria (2021)

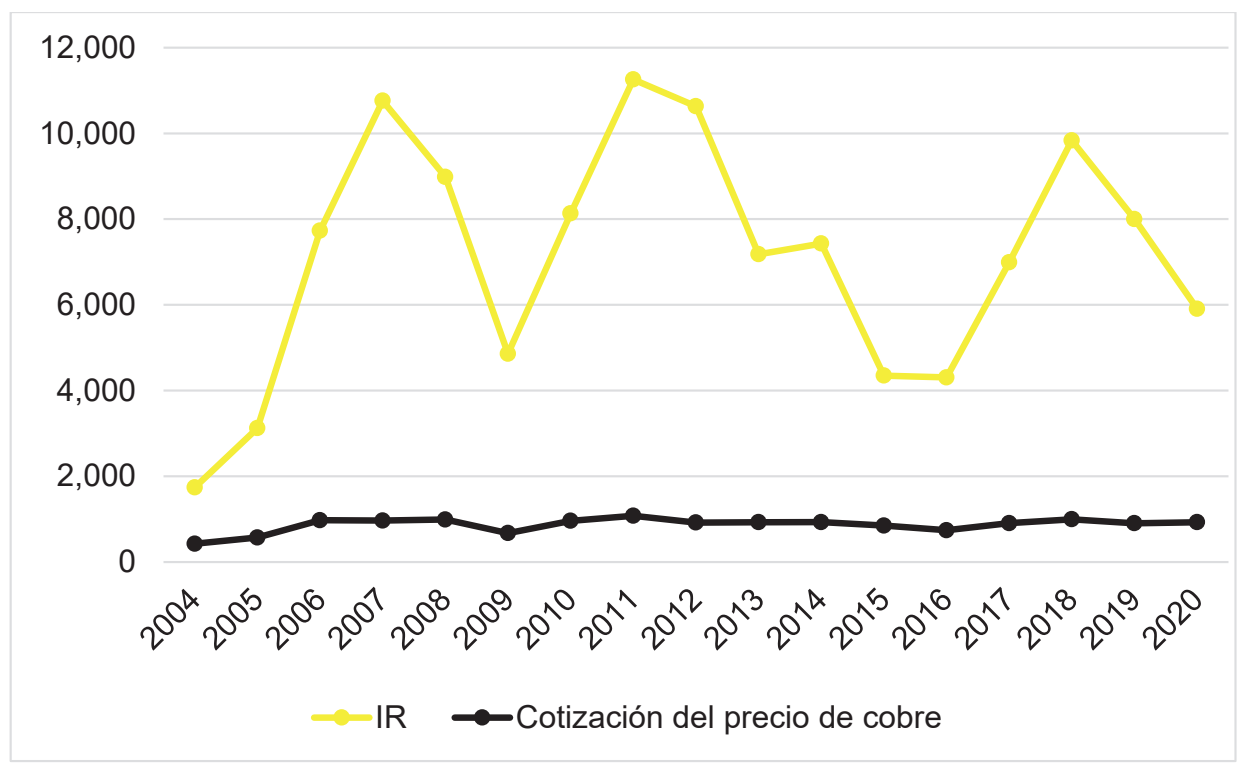

Figura 2. Impuesto a la renta y la cotización del precio de cobre en el periodo 2004-2020 Fuente: Elaboración propia sobre la base de la Superintendencia Nacional de Aduanas y de Administración Tributaria (2021) y London Metal Exchange (2020) 
Al respecto, Hayek (2014) señala que:

El orden construido (...) sostiene que las instituciones humanas solo pueden servir a los objetivos del hombre si han sido deliberadamente diseñadas para estos fines. (...) La misma existencia de esas instituciones sería prueba de que ha sido creada para un fin, y siempre que hay que remodelar la sociedad y sus instituciones todos nuestros actos tienen que ser guiados por fines conocidos (...). El orden espontáneo (...), en gran medida, respondía a un proceso inicialmente denominado "crecimiento" y luego "evolución" (...). (p. 26)

De lo anteriormente señalado, resulta importante resaltar que los impuestos pertenecen a un orden construido, y la cotización internacional del precio del cobre es producto de los intercambios que se realizan en los mercados internacionales que por su naturaleza constituye un sistema de precios que se encuentra en función de la oferta y demanda, estableciendo así, un orden espontáneo.

En línea con lo mencionado, el comparativo que se presenta en la Tabla 1 permite establecer las principales diferencias entre el orden espontáneo y el orden construido, los cuales ayudan a comprender el comportamiento de la economía y sobre todo cómo esta se ve afectada por las acciones de agentes internos y externos.

Desde tal perspectiva, es necesario exponer lo desarrollado por la teoría neoinstitucionalista, ya que esta última se enfoca en el desempeño de la economía bajo reglas de juego informales y formales, también llamadas instituciones, las cuales, según North (1993) “ ¿son formales o informales las instituciones? Puede ser una u otra cosa, pero yo estoy interesado tanto en limitaciones formales, por ejemplo, normas que idean los humanos, como en limitaciones informales tales como acuerdos y códigos de conducta" (p. 14).
De ahí que resulta trascendental comprender la importancia de los impuestos a través de las instituciones formales, ya que resultan ser una pieza fundamental en el desempeño económico del sector minero enfocado en sus tres pilares: sociedad, empresas y Estado. Por lo que, la debilidad de las reglas de juego termina generando incertidumbre. Como refiere North (1993), "las instituciones reducen la incertidumbre estableciendo una estructura más estable, asimismo es de vital importancia definir la diferencia entre las instituciones y organismos" (p. 16).

Por lo tanto, reducir la incertidumbre en el comportamiento de la RIRE y la cotización internacional del precio del cobre ayuda a generar mejores estrategias para mitigar el potencial impacto que podría generar la volatilidad de los precios en dicho impuesto del sector minero. Incluso, resulta trascedente comprender de qué manera se manifiesta el mercado internacional. Para tal efecto, Mendoza (2010) precisa que:

En el mercado internacional coexisten y se relacionan dos tipos de países: los exportadores y los importadores. No es posible que en una determinada mercancía todos los países sean exportadores netos o importadores netos. Si hay países que exportan, ello es porque hay otros que lo importan. Visto de otra manera, no es posible que todos los países sean superavitarios en el comercio de un bien; si los hay, es porque también existen países deficitarios. Los países superavitarios en una mercancía son los exportadores, en tanto que los deficitarios, los importadores. (p. 138)

De lo expuesto, se puede concluir que la cantidad pactada y el precio internacional del cobre se determinan sobre las interacciones constantes de las exportaciones (ofertantes) e importaciones (demandantes). En efecto:

Tabla 1

Comparativo entre el orden espontáneo y orden construido

\begin{tabular}{cc}
\hline Orden espontáneo & Orden construido \\
\hline Orden que se autogenera & Orden que se construye \\
Acciones humanas no intencionadas & Proviene de planes intencionados \\
Carece de finalidad concreta & Tiene fines concretos \\
Racionalismo evolucionista & Racionalismo constructivista \\
"Cosmos" (evolución) & "Taxis" (artificialmente) \\
\hline
\end{tabular}

Fuente: Elaboración propia sobre la base de Rodríguez (2016) 
Los aranceles y las diversas formas de cuotas de importación prohíben, parcial o totalmente, la competencia geográfica para diversos productos (...). También perjudican a las empresas extranjeras más eficientes y a los consumidores en todas las áreas, a quienes se les impide gozar de las ventajas de la especialización geográfica. En un mercado libre, los mejores recursos tienden a asignarse a sus ubicaciones más productivas (...). (Rothbard, 2015, pp. 55-56)

Por lo tanto, resulta coherente que los impuestos (aranceles) al ser parte de un orden construido y no ser eficientes terminan siendo perjudiciales para la economía nacional. Siendo así, se plantea la siguiente pregunta de investigación: ¿Existe relación positiva entre la cotización internacional del precio del cobre y la RIRE del sector minero peruano durante el periodo 2004-2020? De modo que, la hipótesis se formula en los siguientes términos: "Existe relación positiva entre la cotización internacional del precio del cobre y la RIRE del sector minero peruano durante el periodo 2004-2020".

\section{MATERIAL Y MÉTODOS}

El diseño del estudio fue no experimental, retrospectivo, transversal y analítico, con enfoque cuantitativo, y de alcance descriptivo y correlacional. A continuación, se presenta el modelo de Shapiro-Wilk para determinar si la hipótesis proviene de una distribución normal o no:

$$
W=\frac{\sum_{\mathrm{i}=1}^{\mathrm{n}} a_{i} x_{(i)}}{\sum_{\mathrm{i}=1}^{\mathrm{n}}\left(x_{i}-\bar{x}\right)^{2}}
$$

Donde:

$X_{(i)}$ : Número que ocupa la i-ésima posición la cotización internacional del precio del cobre.

$\bar{x}:$ Media muestral.

El valor de W puede oscilar entre 0 y 1 .

Asimismo, se presenta el modelo de correlación de Pearson para determinar si las variables de la hipótesis mantienen una correlación positiva o negativa.

$$
W=\frac{\sum_{\mathrm{i}=1}^{\mathrm{n}}\left(x_{i}-\bar{x}\right)\left(y_{i}-\bar{y}\right)}{\sqrt{\sum_{i=1}^{n}\left(x_{i}-\bar{x}\right)^{2}} \sqrt{\sum_{i=1}^{n}\left(y_{i}-\bar{y}\right)^{2}}}
$$

Donde:

$x, y_{i}$ Puntos muestrales de la recaudación y la cotización internacional del precio del cobre

$\bar{x}:$ Media muestral
A su vez, cabe precisar que la correlación varía en el intervalo de $[-1,1]$, estableciéndose así, el sentido de la relación:

- $\quad$ Si $r=1$, la correlación es positiva perfecta

- $\quad$ Si $0<\mathrm{r}<1$, la correlación es positiva

- $\quad$ Si $r=0$, no existe relación lineal

- $\quad \mathrm{Si}-1<\mathrm{r}<0$, la correlación es negativa

- $\quad$ Si $r=-1$, la correlación es negativa perfecta

Dentro de este marco, se realiza el análisis de regresión lineal simple para establecer el efecto de la cotización internacional del precio del cobre en la RIRE. Al respecto, Córdova (2003) señala que "para la regresión lineal debe contarse con pares de valores $\left(\mathrm{x}_{1}, \mathrm{y}_{1}\right),(\ldots)\left(\mathrm{x}_{\mathrm{n}}, \mathrm{y}_{\mathrm{n}}\right)$ de una variable bidimensional $\left(\mathrm{X}_{\text {Cotización }}, \mathrm{Y}_{\text {Recaudación }}\right)$. Para lo cual, la regresión lineal simple consiste en determinar la ecuación de la recta” (p. 86). Tal como se muestra a continuación:

$$
\mathrm{Y}=\mathrm{a}+\mathrm{bx}
$$

Por lo tanto, la regresión simple ayuda a predecir o estimar "Y" (variable dependiente) a partir de "X" (variable independiente), siempre que se determina los valores de "a" y "b" a partir de los datos de la muestra. Por otro lado, resulta importa precisar que la regresión lineal solo modela las relaciones entre variables dependientes e independientes lineales, lo que supone que dicho método resulte ser muy sensible a las anomalías de los datos.

\section{RESULTADOS}

En la Figura 2 descrita inicialmente, se estableció de manera empírica que la RIRE del sector minero se ve influenciada por el precio internacional del cobre. Por lo tanto, con la finalidad de verificar que las variables mantienen una distribución normal, se procedió aplicar la prueba de normalidad con un nivel de significancia (error tolerable) del 1\%, para lo cual se formularon las siguientes hipótesis:

H1 (H. alterna del investigador): La variable aleatoria es distinta a la distribución normal.

H0 (H. nula): La variable aleatoria tiene distribución normal.

Conforme con lo antes expuesto, se logró verificar que las variables objeto de estudio por el periodo 2004-2020, sí mantienen una distribución normal, ya que el nivel de significancia fue mayor y/o igual al 1\%, tal como se puede observar en la Tabla 2. Por lo tanto, es posible aplicar pruebas estadísticas paramétricas. 
Nótese que, en adición a lo antes señalado, es posible aplicar pruebas estadísticas paramétricas, para lo cual se utilizará la prueba de hipótesis con un nivel de significancia (error tolerable) del 1\%, formulando las siguientes hipótesis:

H1 (H. alterna del investigador): Existe relación positiva y muy fuerte entre la cotización internacional del precio del cobre y la RIRE del sector minero correspondiente al periodo 2004-2020.

H0 (H. nula): No existe relación positiva y muy fuerte entre la cotización internacional del precio del cobre y la RIRE del sector minero correspondiente al periodo 2004-2020.

Finalmente, el nivel de significancia fue 0,000006 tal como se observa en la Tabla 3, por lo tanto, el error es tolerable debido a que fue menor al 1\%. En efecto, se acepta la $\mathrm{H} 1$ y se rechaza la $\mathrm{H} 0$, por lo que se concluyó que la cotización internacional del precio del cobre y la RIRE del sector minero están linealmente relacionadas entre sí. Sin perjuicio de lo expuesto, la estimación de la ecuación de regresión lineal también ayudó a plantear supuestos y proyecciones, ello con la finalidad de evaluar el potencial impacto de la volatilidad del precio del cobre en la RIRE del sector minero para lo cual se determinó la siguiente ecuación de regresión lineal, tal como se observa en la Tabla 4 y Figura 3.

Donde:

$$
F(x)=-5,220+14,23(x)
$$

$\mathrm{F}(\mathrm{x})=\mathrm{RIRE}$ del sector minero peruano

$\mathrm{X}=$ Variante de la cotización internacional del precio del cobre

Tabla 2

Prueba de normalidad de Shapiro-Wilk

\begin{tabular}{lccc}
\hline Descripción & Estadístico & gl & Significancia \\
\hline Recaudación del impuesto a la renta empresarial & 0,965 & 17 & 0,729 \\
Cotización internacional del precio del cobre & 0,826 & 17 & 0,010 \\
\hline
\end{tabular}

Fuente: Elaboración propia sobre la base de la Superintendencia Nacional de Aduanas y de Administración Tributaria (2021) y London Metal Exchange (2020)

Tabla 3

Correlación entre la cotización internacional del precio del cobre y la RIRE

\begin{tabular}{lccc}
\hline Variables & Descripción & RIRE & Cotización internacional del precio del cobre \\
\hline \multirow{2}{*}{$\begin{array}{l}\text { Cotización internacional del precio } \\
\text { del cobre }(\mathrm{X})\end{array}$} & Correlación de Perason & 0,869 & 1 \\
& Sig. (Bilateral) & 0,000006 & 17 \\
& $\mathrm{~N}$ & 17 & 0,869 \\
RIRE $(\mathrm{Y})$ & Correlación de Perason & 1 & 0,000006 \\
& Sig. (Bilateral) & & 17 \\
\hline
\end{tabular}

Fuente: Elaboración propia sobre la base de la Superintendencia Nacional de Aduanas y de Administración Tributaria (2021) y London Metal Exchange (2020)

Tabla 4

Ecuación de regresión lineal

\begin{tabular}{cccccc}
\hline & \multicolumn{3}{c}{ Coeficientes } & & \\
\hline Modelo & B & Desv. Error & Beta & t & Sig. \\
(Constante) & $-5,219,730$ & $1,846,273$ & & $-2,827$ & 0,013 \\
Cotización internacional del precio del cobre & 14,229 & 2,090 & 0,869 & 6,808 & 0,000006 \\
\hline
\end{tabular}

Fuente: Elaboración propia sobre la base de la Superintendencia Nacional de Aduanas y de Administración Tributaria (2021) y London Metal Exchange (2020) 


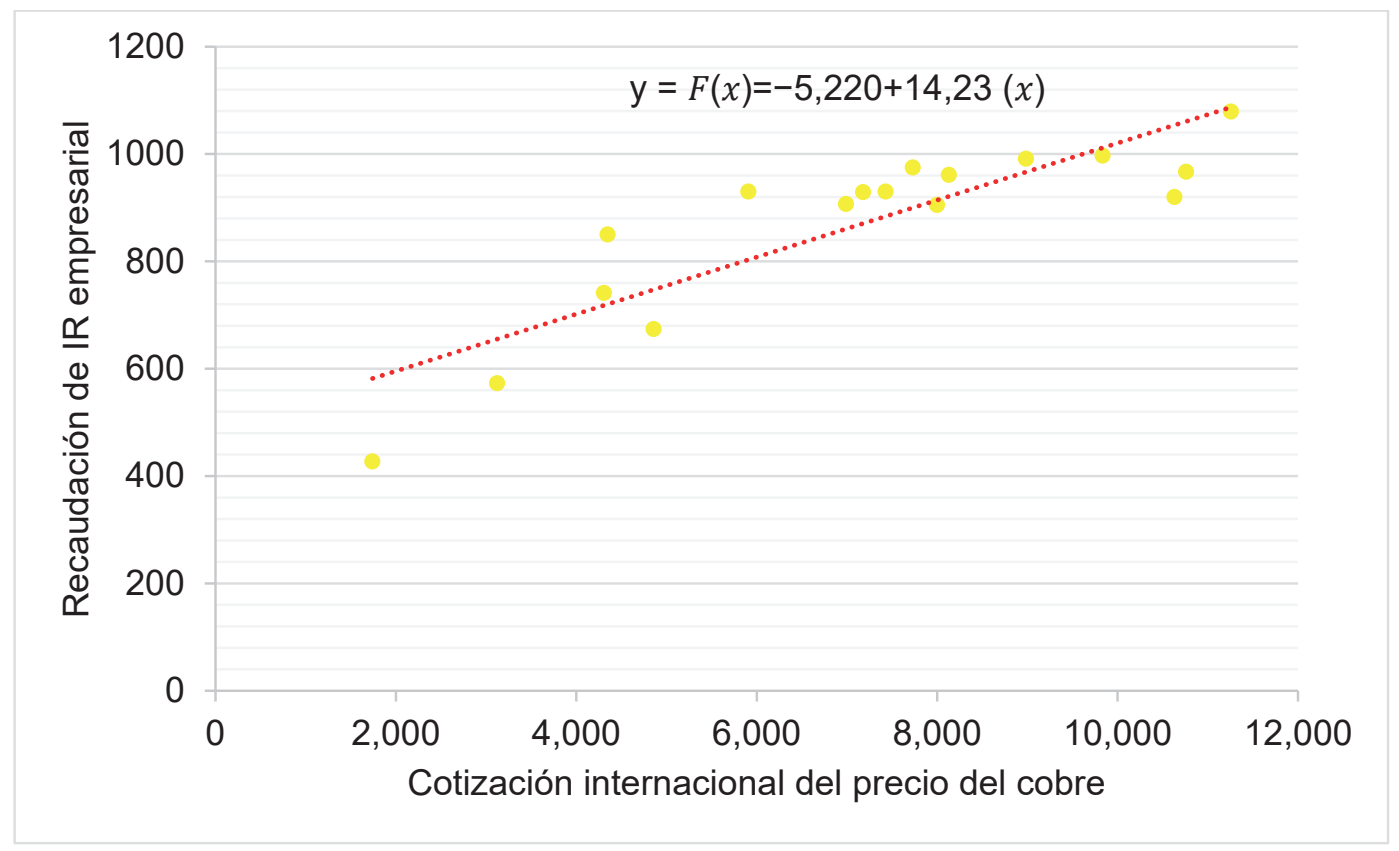

Figura 3. Dispersión lineal de la cotización del precio del cobre del perido 2004-2020

Fuente: Elaboración propia sobre la base de la Superintendencia Nacional de Aduanas y de Administración Tributaria (2021) y London Metal Exchange (2020)

\section{DISCUSIÓN}

Existe relación positiva y considerable entre la cotización internacional del precio del cobre y la RIRE del sector minero peruano correspondiente al periodo 2004-2020, tal como se muestra en la Tabla 3, siendo la correlación entre ambas variables de $86,9 \%$. Así, el grado de variación de la variable dependiente respecto de la variable independiente es del $75,52 \%$.

Esto resulta sumamente importante, ya que complementa al estudio realizado por el Ministerio de Energía y Minas (2020), en donde este último precisó que los principales productos metálicos analizados mejoraron su cotización en los siguientes porcentajes:

En contraste a diciembre de 2019, los principales productos metálicos analizados mejoraron su cotización de la siguiente manera: cobre $(+27,6 \%)$, oro $(+25,4 \%)$, zinc $(+22,4 \%)$, plata $(+45,2 \%)$, plomo $(+6,3 \%)$ y hierro $(+63,5 \%)$, evidenciando una recuperación de la demanda de estos minerales a consecuencia de la reactivación de las actividades fabriles y tecnológicas en los principales países industriales. Mientras que, comparando las cotizaciones promedio de diciembre con los obtenidos al inicio de año (mes de enero), tenemos que el cobre $(+28,5 \%)$, oro $(+18,9 \%)$, zinc $(+18,3 \%)$, plata $(+38,5 \%)$, plomo $(+4,9 \%)$ y hierro $(+58,3 \%)$ experimentaron notorios incrementos en sus precios (...). (p. 13)

De este modo, se resalta diversos factores que inciden en la cotización del cobre; entre los cuales, destaca la aprobación de estímulos fiscales estadounidense, la acelerada recuperación económica de China y el descenso de existencias de cobre, siendo favorable para la situación extractiva económica del país, que se caracteriza por la exportación de materias primas.

Por otro lado, el Estado debe considerar plantear medidas que ayuden a planificar situaciones estratégicas de política fiscal, ya que el impacto de la volatilidad del precio del cobre puede ser dual (a favor o en contra); por lo tanto, deben diseñarse y aplicarse medidas que ayuden a mitigar el efecto adverso en la RIRE y su impacto en la economía nacional.

Finalmente, la investigación fija un precedente para futuros estudios sobre la relación entre la cotización internacional del precio del cobre y la RIRE, debido a que la literatura actual solo se enfoca en el análisis de la cotización de precios bajo un ámbito de producción, inversiones crecimiento económico, y no se analiza de manera específica el efecto que podría generar en la recaudación fiscal. Por ello, en complemento al presente estudio, existen diversas 
preguntas que podrían ser absueltas en futuras investigaciones: ¿Es posible establecer modelos económicos que permita estimar las proyecciones de la cotización internacional del precio del oro, zinc, plata, plomo, hierro y la RIRE del sector minero?, ¿en qué medida influyen en la oferta internacional los niveles de producción de minerales de la empresas establecidas en el Perú?, ¿en qué medida podría impactar la volatilidad de los demás minerales en la RIRE? y ¿en qué medida podría impactar la volatilidad de los demás minerales en la recaudación de otro impuestos?

\section{REFERENCIAS}

Confederación Nacional de Instituciones Empresariales Privadas. (Junio de 2021). La minería formal aporta el 14\% del PBI nacional. Recuperado de https://www. confiep.org.pe/confiep-tv/mineria-formal-aporta-al14-del-pbi-nacional/

Córdova, M. (2003). Estadística, descriptiva e inferencial. Lima: Moshera S.R.L.

De la Cuba, M., \& Ferreyra, J. (2021). Evolución del precio del cobre durante el episodio del COVID-19: Un análisis comparativo. Moneda, (183), 32-37. Recuperado del sitio de Internet del Banco Central de Reserva del Perú: https://www.bcrp.gob.pe/publicaciones/revista-moneda/revista-moneda-183.html

Gestión. (20 de agosto de 2020). Minería puede ser el salvavidas para la economía peruana en recesión. Recuperado de https://gestion.pe/

Hayek, F. A. (2014). Derecho, legislación y libertad (2. ${ }^{\mathrm{a}}$ ed.). Madrid: Unión Editorial.

Instituto Peruano de Economía. (28 de junio de 2021). Cómo impacta la minería en la productividad de Perú. Recuperado de https://www.ipe.org.pe/portal/ipe-como-impacta-la-mineria-en-la-productividad-de-peru/
London Metal Exchange. (2020). LME Copper. Trading day summary. Recuperado de https://www.lme.com/ en-GB/Metals/Non-ferrous/Copper\#tabIndex $=0$

Mendoza, L. J. (2010). Economía aplicada. Lima: Editorial Universitaria UNAC.

Ministerio de Energía y Minas. (2020). La minería peruana en modo COVID-19. Recuperado de http://www. minem.gob.pe/minem/archivos/file/Mineria/PUBLICACIONES/VARIABLES/2020/BEMMAR20.pdf

North, D. C. (1993). Instituciones, cambio institucional y desempeño económico. México D.F.: Editorial Fondo de Cultura Económica.

Peñaranda, C. (19 de agosto de 2019). La minería y su aporte económico al Perú. Recuperado del sitio de Internet de la Cámara de Comercio de Lima: https://www.camaralima.org.pe/articulos-iedep-en-la-revista/

Rodríguez, V. (2016). Libertad económica y bienestar individual en Perú. Quipukamayoc, 24(46), 25-36. https://doi.org/10.15381/quipu.v24i46.13206

Rothbard, M. N. (2015). Poder y mercado. El gobierno y la economía. Madrid: Unión Editorial.

Superintendencia Nacional de Aduanas y de Administración Tributaria. (2021). Información Tributaria. Ingresos Recaudados. Recuperado de https://www.sunat. gob.pe/estadisticasestudios/ingresos-recaudados.html 